\title{
Human body measurement system based on RGB-D camera using
}

\author{
Alexander V. Fisunov*, and Victoria B. Gnezdilova \\ Don State Technical University, 346500 Shakhty, Russia
}

\begin{abstract}
This paper proposes a non-contact method for obtaining dimensional features of a person using an RGB-D camera and analysing a 3D model of a human model constructed using such a camera.
\end{abstract}

\section{The statement of the problem}

At the first stage, when designing clothes, it is necessary to get dimensional features from the figure of the person for whom the clothes are made. As a rule, in classical methods of designing clothes, the getting of dimensional features is carried out by a contact method, with the help of anthropometric instruments and devices, such as a metal anthropometer of Martin system, measurement tape, a large thick calipers, etc. However, when designing adaptive clothing for people with disabilities (PWD), the use of contact method is impossible, since this method involves the getting features from the human figure in the "standing" position without assistance and special devices.

To solve this problem, it is possible to use non-contact methods for getting dimensional features from the figure of the PWD, but existing solutions involve the use of bulky and expensive equipment, and also require the presence of a person in conditions close to laboratory conditions (for example, laser scanning). The use of compact devices for scanning people with disabilities, constructing their 3D models and the availability of tools for measuring these models would significantly simplify the process of non-contact getting of dimensional features from the figure of PWD in the design of adaptive clothing.

\section{Proposed method}

The paper proposes a method for obtaining a 3D polygonal model of a person using RGB-D camera (Microsoft Kinect, Intel RealSense SR300, etc.) with further software processing to obtain the necessary dimensional characteristics of the person on the resulting model.

The portable RGB-D camera is selected to simplify the scanning process and reduce the requirements for the use of bulky equipment in the laboratory. When using cameras such as Microsoft Kinect, scanning can be carried out by anyone at home, sending the resulting 3D model by e-mail for further processing. Figure 1 shows a block diagram of the process of obtaining dimensional characteristics of a person.

\footnotetext{
${ }^{*}$ Corresponding author: fisunov.a.v@gmail.com
} 


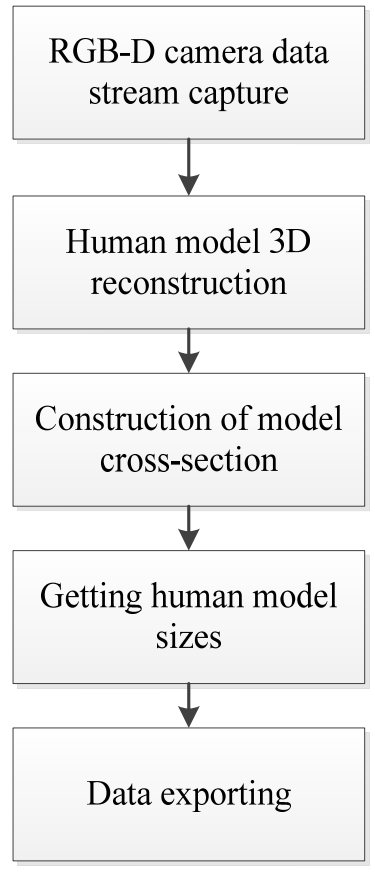

Fig. 1. Algorithm for obtaining dimensional characteristics of a person using RGB-D camera

Obtaining a 3D model of a person is carried out using software based on Kinect Fusion technology [1], which allows you to build a 3D scene in real time at a scale of 1:1. Figure 2 shows an example of the resulting model and the proposed measuring tools.

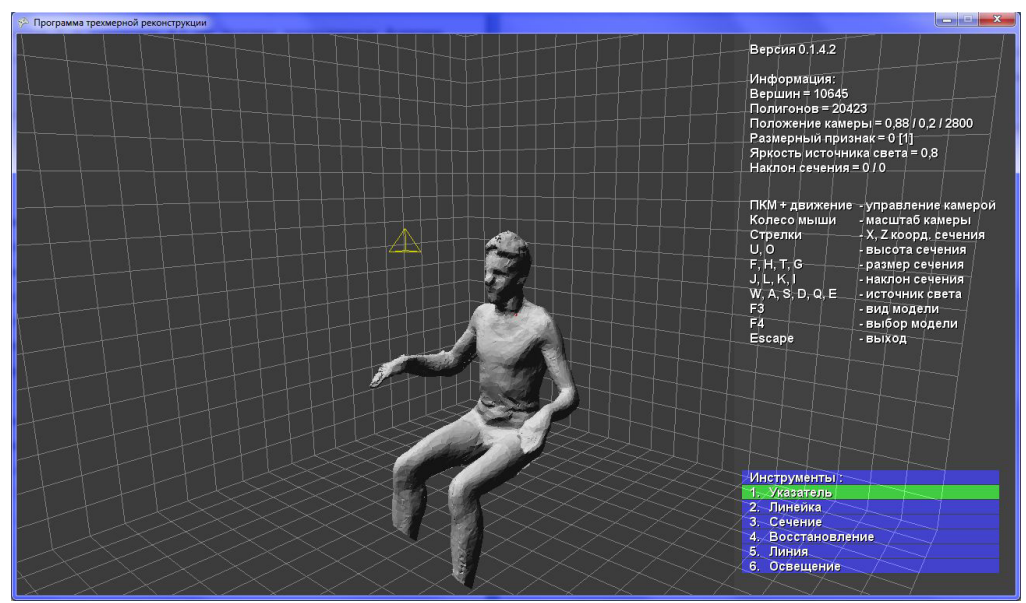

Fig. 2. 3D polygonal model.

To describe the processing operation, it is necessary to explain what a human model is. A polygonal 3D model consists of a set of vertices and edges that connect these vertices. Vertices are joined by polygons, which are usually triangles in computer graphics. To obtain dimensional features of such a model, it is sufficient to construct lines and find the cross section of an object in 3D space.

The measurement process consists of several stages. In the first stage we find the polygons, and the edges that intersect with a given plane of section. For each edge, we 
calculate the point of intersection with the plane, which will result in a set of points on the plane. According to this set, the method Convex Hull $[2,3]$ builds a polygon, which is a section of one of the parts of the body. Figure 3 shows an example of the resulting set of points from the cross section of the object and the result of using the Convex Hull method.

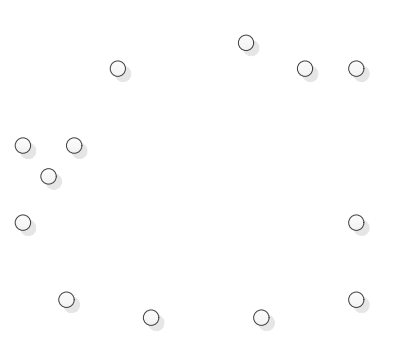

(a)

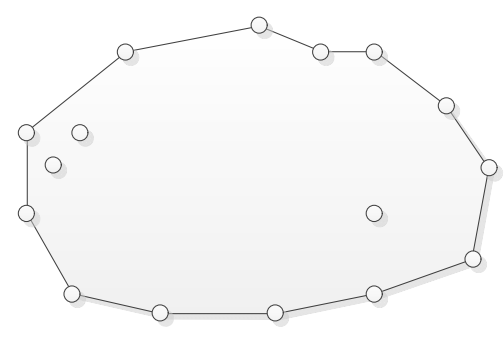

(b)

Fig. 3. An example of the obtained set of points from the section (a) and the construction of a convex hull on this set by the Convex Hull (b) method.

When scanning an object with RGB-D camera, the model may not be perfectly smooth, angular surfaces and outliers may appear (mistakenly found surface points). In reality, the parts of the human body have a smooth shape, so the boundaries of the polygon are smoothed. The smoothing result is shown in figure 4 .

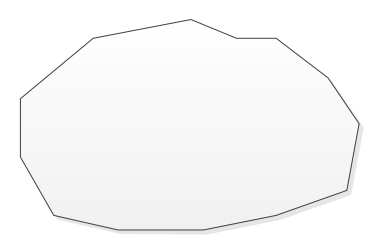

(a)

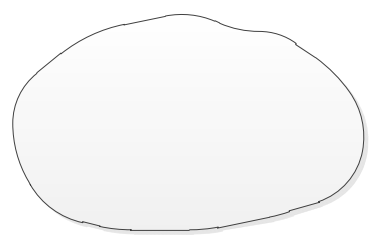

(b)

Fig. 4. Polygon before (a) and after (b) smoothing.

After smoothing the polygon, it remains only determining the length of its boundaries and put it in correspondence with one of the dimensional features.

To build a line in 3D space, you must define two points on the model between which the line passes. To determine the polygons which are the initial and final boundary of a segment, the ray-plane intersection algorithm (Line-Plane Intersection) is used. At figure 5 examples of measurement instruments is presented.

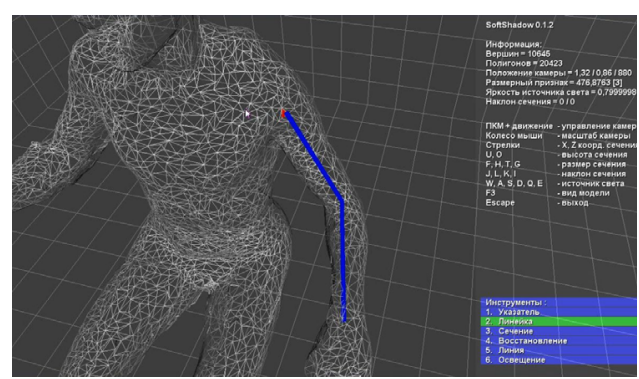

(a)

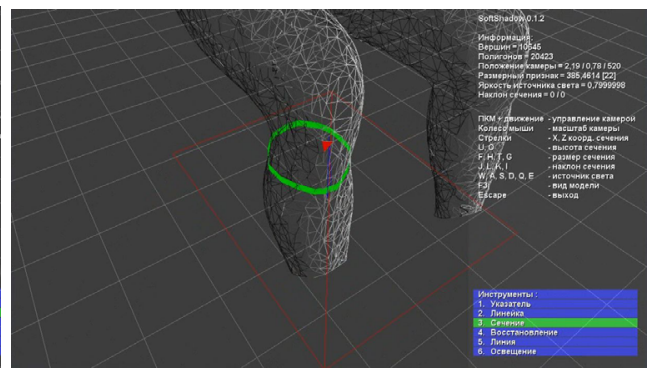

(b)

Fig. 5. Proposed measurement tools for 3D polygonal model (a) line segment tool, (b) cross-section with plane 
Having the ability to find the cross section of an object, build lines between polygons, and determine the size of the resulting segments, you can get all the necessary dimensional features of a person from its polygonal 3D model.

\section{The obtained results}

To test the proposed method we use the camera, RGB-D Microsoft Kinect for obtaining model of several people. Table 1 shows the results for two people.

Table 1. Comparison of dimensional features obtained by contact and proposed non-contact measurement methods

\begin{tabular}{|c|c|c|c|c|c|c|}
\hline $\begin{array}{c}\text { Feature } \\
\text { index }\end{array}$ & $\begin{array}{l}\text { Real feature } \\
\text { size, cm }\end{array}$ & $\begin{array}{c}\text { Feature size } \\
\text { from } 3 \mathrm{~d} \\
\text { model, } \mathrm{cm}\end{array}$ & $\begin{array}{l}\text { Relative } \\
\text { error, \% }\end{array}$ & $\begin{array}{c}\text { Real } \\
\text { feature } \\
\text { size, } \mathrm{cm}\end{array}$ & $\begin{array}{c}\text { Feature size } \\
\text { from } 3 d \\
\text { model, } \mathrm{cm}\end{array}$ & $\begin{array}{l}\text { Relative } \\
\text { error, \% }\end{array}$ \\
\hline 1 & 85,5 & 87,2 & 1,9 & 82,5 & 84,1 & 1,9 \\
\hline 2 & 89 & 91,8 & 3,1 & 86,5 & 88,5 & 2,3 \\
\hline 3 & 88 & 90,2 & 2,5 & 84 & 86,3 & 2,7 \\
\hline 4 & 33,5 & 35,2 & 5,1 & 32,7 & 34,1 & 4,3 \\
\hline 5 & 36,5 & 36,2 & 0,8 & 37 & 38,4 & 3,8 \\
\hline 6 & 26,5 & 28,1 & 6,0 & 28,5 & 29,3 & 2,8 \\
\hline 7 & 15 & 16 & 6,7 & 16 & 17 & 6,3 \\
\hline 8 & 52,5 & 53,8 & 2,5 & 50 & 50,9 & 1,8 \\
\hline 9 & 43,5 & 43 & 1,1 & 41 & 41,4 & 1,0 \\
\hline 10 & 42,5 & 44 & 3,5 & 41,5 & 41,5 & 0,0 \\
\hline 11 & 43 & 44,2 & 2,8 & 43,5 & 41,4 & 4,8 \\
\hline 12 & 20 & 21,3 & 6,5 & 17,5 & 16,7 & 4,6 \\
\hline 13 & 24 & 23 & 4,2 & 23,5 & 22,5 & 4,3 \\
\hline 14 & 60 & 61 & 1,7 & 58,5 & 56,9 & 2,7 \\
\hline 15 & 110 & 107 & 2,7 & 115 & 111,3 & 3,2 \\
\hline 16 & 94,5 & 92,2 & 2,4 & 96 & 94,3 & 1,8 \\
\hline 17 & 77 & 74 & 3,9 & 77 & 73,2 & 4,9 \\
\hline 18 & 30,5 & 28,9 & 5,2 & 25,5 & 26,8 & 5,1 \\
\hline 19 & 28,5 & 30 & 5,3 & 31,5 & 29,8 & 5,4 \\
\hline 20 & 12 & 13,1 & 9,2 & 14,5 & 13,5 & 6,9 \\
\hline Avg & & & 3,8 & & & 3,5 \\
\hline
\end{tabular}

Table 1 shows that the average value of the relative error is no more than 5 percent. If contact methods to get dimensional features can not be applied, such an error is not high. As a possible improvement can be made by reducing the polygon size when building the model and using other filtering methods.

The obtained results, for example, can be exported to CAD for cloth modelling and construction. 


\section{Conclusion}

In the paper, a method is proposed for getting dimensional features from the figure of a person in the "sitting" position using his 3D model, obtained with the help of an RGB-D camera. The results of the study showed that the average value of the relative error is not more than $5 \%$, which meets the requirements of clothing design.

\section{References}

1. S. Izadi, D. Kim, O. Hilliges, D. Molyneaux, R. Newcombe, P.Kohli,... \& A. Fitzgibbon, In. Proc. ACM symp., 559 (2011)

2. T. M. Chan, Discrete \& Computational Geometry, 16(4), 361 (1996)

3. J. Bentley, G. Faust, F. Preparata, ACM, 25, 64 (1982)

4. P.H. Lin, H.K. Chu, T.Y. Lee, IJICIC, 4 (9), 2405 (2008) 